\title{
Women's Employment: Joining Explanations Based on Individual Characteristics and on Contextual Factors
}

\author{
By Angela Cipollone and Carlo D'Ippoliti
}

\section{Introduction}

The dramatic increase in women's activity and employment rates explains a large part of the secular increase in labor supply that took place during the second half of the $20^{\text {th }}$ century in many industrial countries. It constitutes one of the most interesting facts of contemporary economic history and indeed it stimulated an extensive debate. This article takes issue with the mainstream economics explanations of this phenomenon. The mainstream economic theory traditionally approaches the issue of gender differentials in the labor market by means of microeconomic (often game-theoretic) models of the household, ultimately based on gender differences in preferences and/or endowments (sometimes attributed to gender-based discrimination). At the same time, the applied mainstream literature has extensively studied other relevant factors such as the role of education, social policy, macroeconomic conditions, and culture. This literature offers interesting insights on the determinants of women's employment but it suffers from a main limitation; it mostly developed into two related but separate strands. One of these strands deals with individuals' characteristics and how they affect women's labor supply, by usually employing micro-data surveys. The other strand investigates the relevance of the institutional context and public policy (or "macro" factors), by usually analyzing cross-sections or panels of aggregate data. ${ }^{1}$

While each strand of literature recognizes it is linked to the other, they usually ignore or assume as exogenously given the relations, variables, and theories of the other strand. This separation is problematic from an applied point of view because it marks a possibly untenable ceteris paribus hypothesis. This article argues that the ignorance of possible interactions between micro and macro factors American Journal of Economics and Sociology, Vol. 70, No. 3 (July, 2011).

(C) 2011 American Journal of Economics and Sociology, Inc. 
may produce sensible biases in the applied estimates, for two reasons. ${ }^{2}$ On the one hand, if the impact of individual characteristics depends on the environment, the individual estimates of any regression that do not control for these interactions could at best be considered as valid only on average and for the specific setting in which they are computed. On the other hand, the macro-level variables may not only exert a direct effect on women's employment, but also an indirect one, by modifying the impact of micro variables. The resulting estimates of a regression that ignores these interactions may thus suffer from a missing variable bias and lead to biased estimates of the impact of the micro variables.

In the few papers that explicitly deal with this issue, the problem is usually treated by means of a difference-in-difference approach, that is, by employing dummy-variable interaction terms between micro and macro variables. However, this method is not feasible if the environment affects individual values by means of several factors simultaneously, again for two reasons. First, observations may be clustered by region; therefore, the error term of the regression is not independently and identically distributed. Second, accounting for the simultaneous direct and indirect impact of many environmental factors may require an excessive number of dummy variables. This article proposes the use of multilevel analysis as a more direct and rigorous way to simultaneously estimate the impact of micro and macro factors within a unified regression model. This method allows one to consider a wider set of determinants of women's employment than is possible with a standard regression analysis, for example, the simultaneous role of education and social policy. However, due to utmost computational requirements, the use of multilevel analysis suggests the prior application of a multivariate analysis to summarize a great number of macro variables into a small set of synthetic macro indices.

This article aims to contribute to the literature on understanding women's work in two ways. On the one hand, we criticize the standard applied mainstream literature by showing the existence of significant interactions between the micro and macro levels. Consequently, our estimates should be regarded as superior to those developed within single-level models (models that only consider micro or 
macro variables). On the other hand, we estimate the relative weight of individual and macro variables in determining women's employment in Italy at different points in history. By doing so, we provide a coherent explanation of the secular increase of women's employment in Italy.

The article focuses on the individual-level probability of being employed, by developing a unique dataset of individual and regional observations for selected years between the 1970s and the 2000s. ${ }^{3}$ We consider Italy to be an internationally relevant case study because it currently exhibits the second lowest women's employment rate in the European Union after Malta and some of the highest territorial heterogeneity among European countries. This heterogeneity spans from the cultural and political environment to economic development, social institutions, past and current policies, and it is partly the result of a very late period of national unification. Thus, the comparison of Italy's regions provides a good framework to estimate the impact of the variation in several contextual variables simultaneously.

Our results allow us to partly reject some common beliefs established in the mentioned mainstream economic literature. For example, we find an ambiguous role of macroeconomic growth, while the market provision of services is found to negatively affect women's employment. Throughout the period, we find a role for education even greater than what is usually estimated, as well as an enduring relevance of unpaid labor and family-related constraints. Concerning macro variables, we find that civic development in particular exerts a positive impact, increasing over time. Overall, although microeconomic characteristics are found to be the most relevant determinants of employment, we find that the impacts of household arrangements and contextual factors are large as well.

The rest of the article is organized as follows. In the next section, we summarize the applied mainstream economics literature on women's employment. The third section presents some data on Italy, distinguishing between the micro and macro levels. The next section clarifies the research question and the hypotheses to be tested. The following section presents the significant results of the multilevel estimations, and the last section discusses the conclusion. 


\section{The Mainstream Economic Literature on Women's Employment}

By focusing on individual characteristics, the first of the two mentioned streams of literature has often pointed out education as the major factor affecting women's employment. Within mainstream economic theory, education is assumed to affect potential wages, and in turn, labor market supply and fertility decisions (Gustafsson and Kenjoh 2008). Education gained increasing attention in explaining the likelihood of women to be employed because of a substantial incoherence between the standard Beckerian models of the gender division of labor against the observed trends. Indeed, the theory of households' allocation of time developed after Becker (1965) implies that an increase in husbands' income would induce women to consume additional non-market time, thereby reducing their probability to be involved in market work. Thus, on the basis of this model we should have faced a historical reduction in women's labor supply. To cope with this predictive failure, the mainstream literature has often argued that this negative income effect may in fact have been mitigated and possibly reversed by the increased educational attainment of women coupled with the phenomenon of mating by education (Eckstein and Lifshitz 2009).

Next to education, housework burdens and family-related responsibilities are usually taken into consideration. Several works (for example, Goodpaster 2010; Leigh 2010; Munasinghe et al. 2008) found that these commitments increase women's reservation wage and in turn decrease the likelihood for low-earning women to enter paid employment. Thus, policies alleviating the financial burden of childrearing were shown to exert a positive impact on women's labor supply (for example, see Sánchez-Mangas and Sánchez-Marcos 2008).

Individual and household characteristics are traditionally the building blocks of mainstream economics, due to its commitment to methodological individualism. However, in applied works, several new topics began to be investigated with increasing attention and detail. On the one hand, the empirical evidence called for such an improvement. For example, several papers found that the relevance of household-related responsibilities to women's employment exhibits country-specific patterns (Bardasi and Gornick 2003; Jaumotte 2003; 
Del Boca et al. 2009). On the other hand, the imperialistic impetus of mainstream economics allows for a greater freedom in the applied fields to devote attention to some factors traditionally considered within the realm of sociology.

Indeed, the second of the two mentioned streams of literature, that focusing on macro characteristics, found, for example, that countries with more generous fiscal and social policies for the support of working mothers are characterized by a greater labor market inclusion of women. The set of relevant public policies may include parental leave schemes (see, for example, Simonazzi 2006), the structure of social benefits and the tax system (OECD 2004), or the availability and quality of public childcare facilities (Berlinski and Galiani 2007; Powell 1998; Anderson and Levine 1999; Del Boca 2002; Attanasio et al. 2008). An interesting theme within this literature is the comparison between the effectiveness of monetary support as opposed to the public provision of services. For example, Apps and Rees (2004) find that among the OECD countries, those supporting motherhood through childcare facilities rather than child benefits tend to exhibit both higher rates of women's labor supply and higher fertility rates (thus, providing evidence against the trade-off between fertility and women's employment rates predicted by models à la Becker). Some works point out that women's labor supply may be more elastic to the quantity of publicly provided childcare facilities than to their price (Lundin et al. 2008).

Next to social policies, the role played by social and cultural norms is gaining increasing attention. Works in this area show that countries with more liberal attitudes towards gender roles, higher work orientation of women, and a higher acceptance towards working mothers of young children, are also characterized by higher women's employment rates (for example, see Algan and Cahuc 2007; Fernandez 2007; Fortin 2005; Giavazzi et al. 2009). Following the literature on statistical discrimination developed after Becker, gender-based discrimination holds a prominent role within the literature on the impact of culture and gender roles on women's employment (see, for instance, Goldin 2002, 2006).

Finally, the relevance of (macro)economic conditions in facilitating or hindering women's employment was investigated mainly with 
respect to the fairly diffused hypothesis that women's labor supply would be more elastic to wage increases than men's. The most debated issues are the supposed emergence of a trade-off between women's employment and the gender pay gap, and of crosscountry positive correlations between women's employment and (i) gender occupational segregation, (ii) the extension of the service sector, and (iii) aggregate GDP growth (for a review of this evidence, see Boeri et al. 2005; Fuchs 1980; Galor and Weil 1996; Goldin 1990). The recent debate on the gender impact of the current financial and economic crisis represents an interesting application of these issues (see, for example, Antonopoulos 2009; ILO 2009; Smith 2009). ${ }^{4}$

As mentioned, these two streams of literature appear to be related, although they are substantially separate. They are mostly based on different types of datasets (microeconomic surveys are used within the stream of literature dealing with the role of individual characteristics, macroeconomic data within the one dealing with the role of institutional characteristics) and usually consider the issues raised by the other stream only in their theoretical accounts, with little impact on empirical practice. In the face of this inconvenience, Del Boca and Sauer (2009) offer an indirect solution. They compare longitudinal micro-data from Italy, France, and Spain, to infer the role of public policies on women's employment and fertility decisions. First they estimate the role of individual and household variables, and then indirectly infer the role of contextual factors. Specifically, they assume that these three countries are similar with respect to culture and gender roles and they run separate estimations for each country. Then Del Boca and Sauer compare the coefficients (that is, the average impact) of an individual characteristic in the three countries, and interpret the emerging differences between the coefficients in the three countries as the result of differences in their social policies. This procedure, however, suffers from a double limitation. On the one hand, it does not allow the authors to disentangle the role of the several macro variables that could simultaneously vary across the three countries. On the other hand, it rests on untested hypotheses concerning the impact of individual and macro variables, and in particular, their interaction. 
By extending the view to a wider set of theoretical approaches, the sociological literature offers relevant insights on the usefulness of methods other than regression analysis. Crucially, it also highlights how multilevel modeling (also known as hierarchical linear modeling or micro-macro analysis) may profitably be employed in the quantitative study of women's work (van der Lippe and van Dijk 2002). This method has thus far has been mainly applied to the study of the household division of unpaid work (for example, Fuwa 2004; Fuwa and Cohen 2007; González et al. 2009; Knudsen and Wærness 2007; Kunovich and Kunovich 2008; Stier and Lewin-Epstein 2007; Voicu et al. 2008) rather than of women's employment in the market (as, for example, done by van Damme et al. 2008).

\section{Recent Dynamics in Italy}

Italy constitutes a very convenient case study to investigate the impact of different local conditions from a historical perspective. From the geographical point of view, Italy exhibits the second-lowest women's employment rate in the European Union and the highest index of regional heterogeneity, as measured by the standard deviation across regions in a number of macroeconomic variables including women's employment rate. ${ }^{5}$ From a historical point of view, the Italian case is characterized by a rapid socio-economic development and a very pronounced regional divergence of women's employment rates. As shown in Figure 1, towards the end of the 1970s the substantial territorial heterogeneity implied a 10 percent difference in both men's and women's employment rates between the more advanced regions in the north and those in the south (Italy is composed of 20 administrative regions that are usually grouped into relatively homogeneous geographical areas). Starting from the 1980s, while men's employment exhibited a rough uniform decrease in all regions - up to 10 percent at the end of the period-women's employment rates diverged substantially. In the north and the center they increased (by more than 10 percent), while regions from the south lagged well behind the rest of the country.

To analyze these trends we merge a dataset of macro variables with data from the Bank of Italy's Survey of Households' Income and 
Figure 1

Long-Run Evolution of Employment Rates of Women and Men, Italy 1977-2009

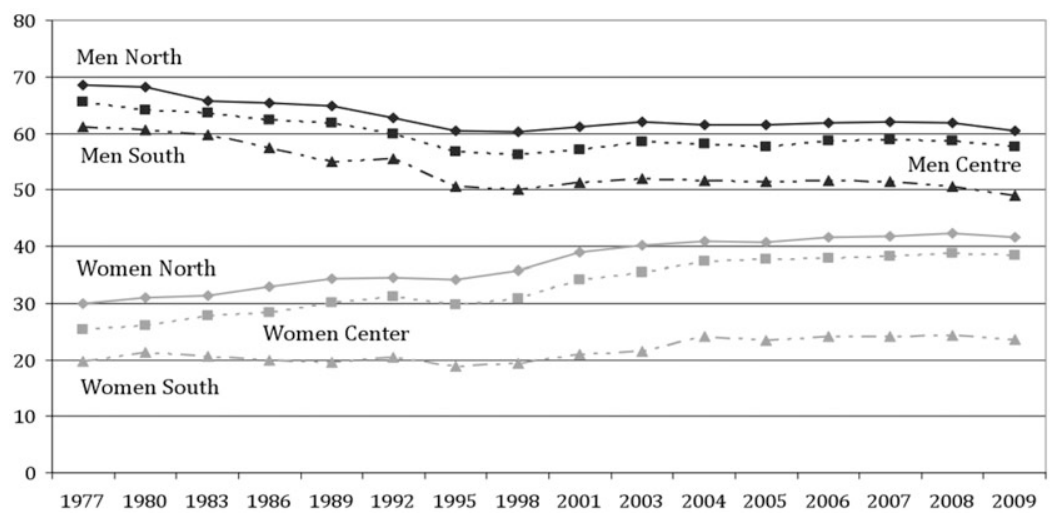

Source: Potestio (2005) and elaboration on ISTAT, Labor Force Survey (various years).

Wealth (SHIW), a representative sample of Italy's population released at irregular time lags. We consider four representative years between the 1970s and the 2000s, namely in 1977, 1986, 1995, and 2004, selected on the basis of availability of microdata. ${ }^{6}$ We consider only individuals of prime-age (between 25 and 54 years old) in order to avoid interference with educational and early-retirement processes and with school-to-work transitions. Our sample is composed of 4,357 individuals for year 1977, of which 2,863 are women, 7,426 for 1986 (2,516 women), 10,350 individuals for 1995 (5,274 women), and 8,716 for 2004 (4,476 women).

In addition, for each year we collect a number of variables from several sources aggregated at the regional level, selecting those more frequently considered in the mainstream economic literature summarized above. The variables selected are listed in column 1 of Table 1. We normalized all variables by their national average, and aggregated some of them into homogeneous indices. This procedure, as well as the subsequent multivariate analysis, is made necessary by the very high computational requirements for the estimation of multilevel models, which suggests the inclusion of a limited number of variables 


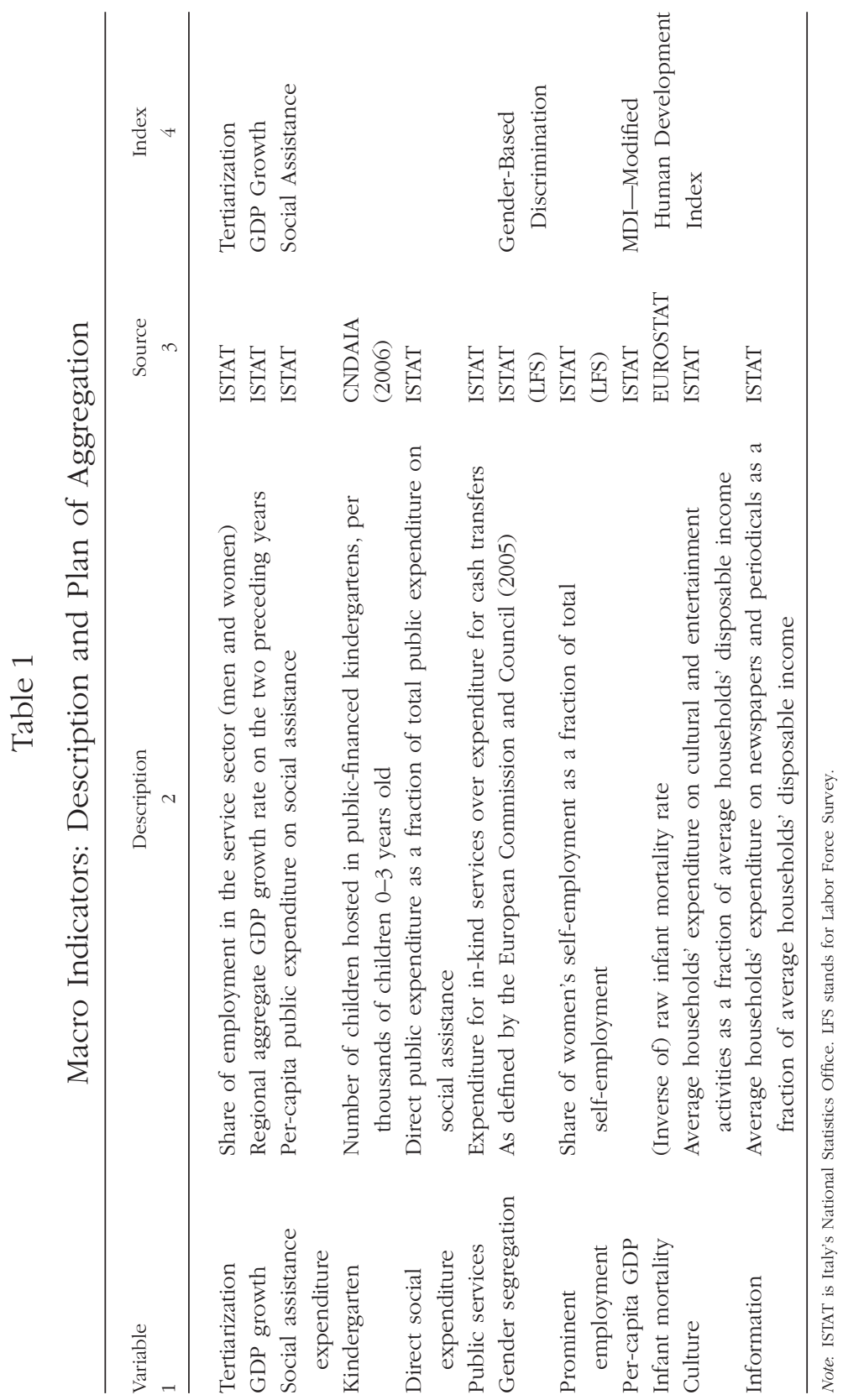


for the higher (macro) level. ${ }^{7}$ However, by first aggregating a number of homogenous indices and then running a principal-component analysis, this research is able to preserve a meaningful economic interpretation of the resulting variables while allowing for the consideration of several different factors within the same analysis. Data over such a long time span and with such a detailed regional focus are very difficult to collect even in European countries, and for a number of variables this study is bound to refer to proxy measures. However, one of the advantages of the use of a multivariate analysis is not only of the help in the selection of variables to be aggregated in order to get a synthetic index, but most notably, to retain in each index only the part of each variable's variance that is correlated with the variance of the other variables that compose the index. Thus, it may be said that the resulting indices convey more indirect information-liable to some disagreement in the interpretation of its economic meaning-but this information is more specific and self-consistent. Thus, even though some of the original variables loosely proxy the measures of interest, by retaining only the part of their variance that is more correlated with the other variables included in the same index, this research makes the best possible use of them and obtains the most reliable final synthetic indicators possible.

To aggregate variables, the first step is to make use of harmonic means in order to get a close measure of the aggregates of interest in their multidimensional nature. In the construction of the indices, harmonic means place more weight on the regions with a more even development of all the relevant variables, and less weight on those exhibiting some disproportionate value in one of the variables (for more details, see Casadio and Palazzi 2004). Descriptive statistics for the original variables and the aggregated indices are reported in Table A1 in Appendix. ${ }^{8}$

As shown in column 4 of Table 1, this study obtains five synthetic indices representing the regional levels of tertiarization, GDP growth, social assistance, gender discrimination, and a modified human development index. The index of tertiarization measures the region's share of employment in the service sector, while GDP growth is the yearly GDP growth in the region, lagged by one year. The nature and extension of publicly-provided social assistance is a proxy of public 
policy aimed at promoting the reconciliation of family responsibilities and market work. It is a compound measure of: per-capita public expenditure on social assistance, the percentage of children hosted in publicly-financed kindergartens, the share of direct social expenditures, and of in-kind expenditures over the total public social expenditure. The discrimination index synthesizes both horizontal (occupational) and vertical gender segregation ${ }^{9}$ in the labor market. In the absence of better data, the latter is proxied by the number of women holding prominent self-employed occupations as a share of the total number of self-employed people in the region. ${ }^{10}$ Finally, our modified index of human development mainly captures the regions' sociocultural development. As shown in Table 1, it differs from the Human Development Index in two respects: aggregation is obtained by harmonic instead of arithmetic mean (as previously mentioned), and some underlying variables are different in order to more accurately catch the relevant differences among Italy's regions while avoiding the inclusion of some variables that will be measured at the individual level (such as educational attainments).

In order to adopt more synthetic macro indicators and to reduce the number of variables to be considered in the analysis, a principalcomponent analysis using the 20 regional observations in the four years was carried out on the five mentioned indices, plus an index of time. The analysis was carried out while keeping common practice (see, for instance, Nardo et al. 2005; Nicoletti et al. 1999; Kline 2004), ${ }^{11}$ and this study obtained three macro indicators that are liable to a straightforward interpretation, as shown in Table 2.

The first factor is highly correlated with our modified index of human development $(+0.88)$ and the public provision of services $(+0.61)$, while it is negatively correlated with the gender discrimination index $(-0.87)$. Due to the specific socioeconomic differences among Italy's regions, and social assistance being an important public policy for reconciliation, this research is inclined to interpret this factor as an index of regional gender-aware civic development (henceforth $\mathrm{CD}$ ). The second factor is negatively correlated with the public provision of social assistance $(-0.41)$, and positively correlated with the extension of the service sector (0.72) and time (0.79). Thus, a plausible interpretation of this factor is that it measures the extent of the private 
Table 2

Results from the Principal-Component Analysis

\begin{tabular}{lccc}
\hline Index & $\begin{array}{c}\text { Civic } \\
\text { Development }\end{array}$ & $\begin{array}{c}\text { Private Provision } \\
\text { of Services }\end{array}$ & $\begin{array}{c}\text { Macroeconomic } \\
\text { Conditions }\end{array}$ \\
\hline Time & & $+0,79$ & \\
Tertiarization & $+0,72$ & $+0,92$ \\
GDP Growth & & $-0,41$ & \\
Social Assistance & +0.61 & & \\
Gender Discrimination & -0.87 & & \\
MDI & +0.88 & & \\
\hline
\end{tabular}

Note: The principal-component analysis was used to extract the factors, using 80 observations for the 20 regions in the four selected years. The rotation of factors was then adopted by means of the varimax method.

Source: Elaboration on various sources (see Table 1).

(market) provision of services to households (henceforth PPS). Finally, the third factor is highly correlated with the lagged regional GDP growth (+0.9), and has a weak negative correlation with tertiarization. As Italy's service sector exhibits on average a lower productivity growth than the manufacturing sector, we interpret this factor as a measure of local macroeconomic conditions (henceforth LMC).

These three indices will be used in the following multilevel analysis to explain cross-regional systematic differences in women's employment rates that cannot be explained by average differences in individual characteristics.

\section{Merging Individual Characteristics with Regional Heterogeneity}

Moving beyond the traditional economic literature, we propose a model of behavior where individual preferences and constraints are mediated by the institutional and economic setting. To explain women's employment, we assume that in the determination of individual employment status factors from different levels (micro and macro) may interact with each other, producing a compound effect. Such interactions would make the separate estimation of the different 
levels of the impact unfeasible under linear regressions, as explained above. We propose a model that allows us to directly estimate the determinants of women's individual employment status, thus disentangling the direct and indirect impacts of both micro and macro variables. The idea of a direct impact is straightforward: both micro and macro variables may be systematically correlated with higher (lower) individual probabilities of being employed. However, an indirect impact may be in place when macro variables modify the impact of individual characteristics on women's employment. For example, the availability of childcare facilities may affect women's employment post motherhood.

Our econometric specification consists of a multi-level analysis based on a probit model of the determinants of employment with observed heterogeneity (that is, explanatory variables) at the individual (first) and the regional (second) levels. We also include unobserved heterogeneity at the regional level, that is, a region-specific systematic deviation that is not accounted for by the three indices developed in the previous section. ${ }^{12}$

Based on the literature reviewed in the second section, we propose three hypotheses concerning the role of macro factors. These hypotheses assert that there is a direct effect of macro factors on the individuals' employment status, meaning that the contextual conditions systematically affect the regions' average employment rates:

$\mathrm{H}_{1}$ : Regions with more advanced policies on equal opportunities and more liberal cultural norms towards gender roles exhibit higher women's employment rates.

$\mathrm{H}_{2}$ : Regions with more progressive social policies, in terms of public services for the reconciliation of work and family life, exhibit higher women's employment rates.

$\mathrm{H}_{3}$ : Regions with higher macroeconomic growth exhibit higher women's employment rates.

To test for the first hypothesis, we measure the effectiveness of policies for gender parity in the labor market by the absence of relevant barriers to entry against women. The two issues of genderbased discrimination and cultural norms are jointly treated because the absence of gender discrimination provides ideological support to a dual-earner household model, and by providing more equal labor 
incomes, it reduces the financial incentive to have only women do all the unpaid work. For its ability to summarize the common components of gender-based discrimination and of the cultural environment with respect to gender, we summarize $\mathrm{H}_{1}$ by the assumption of a positive impact of our index of regional $\mathrm{CD}$.

We frame the second hypothesis in terms of public service in order to indirectly test the associated hypothesis that a commodification of care activities (as summarized by the market provision of such services) is negative for women's employment. Thus, $\mathrm{H}_{2}$ is summarized by the assumption that our index of PPS to households exerts a negative impact on women's employment. By contrast, a positive correlation between the two variables would indicate that the market provision of services is beneficial to women's employment by reducing their care and housework burden, as commonly held by the mainstream literature.

Finally, with the third hypothesis this study tests a widely diffused tenet of the mainstream economics literature, namely that women's labor supply exhibits a higher elasticity to the business cycle than men's. If $\mathrm{H}_{3}$ is correct, our index of LMC will exhibit an impact on women's employment that will be consistently positive and higher than men's.

However, due to the centrality of interaction terms between micro and macro variables in supporting and justifying our approach, we also investigate three further hypotheses to predict how macro variables affect the association between women's individual characteristics and their involvement in market work:

$\mathrm{H}_{4}$ : Macro variables significantly affect the impact of individual and household characteristics on women's employment.

$\mathrm{H}_{5}$ : In regions with more advanced policies on equal opportunities and more liberal cultural norms towards gender roles, paid and unpaid work burdens are more evenly distributed within the household.

$\mathrm{H}_{6}$ : In regions with more progressive social policies, in terms of public services for the reconciliation of work and family life, housework and family-related responsibilities have a weaker effect on women's involvement in market employment.

On the whole, these hypotheses state that the same macro factors mentioned above exert an indirect effect on women's employment 
$\left(\mathrm{H}_{4}\right)$ by affecting the relevance for women of having formed a family $\left(\mathrm{H}_{5}\right)$ or by reducing the constraint of their unpaid work burdens $\left(\mathrm{H}_{6}\right)$.

We capture the negative effect on women's employment arising from joining a household and having to carry out unpaid work, by the impact of a dummy variable- "married"-by which we denote any long-term heterosexual emotional partnership that involves cohabitation. Thus, under $\mathrm{H}_{5}$ we test for a negative interaction between "married" and our index of CD.

We capture the detrimental impact on women's employment arising from unpaid work burdens by analyzing the impact of cohabiting with a person of old-age (above 75 years old) as a proxy of eldercare burden. Thus, under $\mathrm{H}_{6}$ we test for a negative interaction between a dummy variable "old age" and our index of PPS. In the face of the ongoing dramatic development of the aging population in Europe, we deem living with an old-age person as a better proxy of care burdens than having children, for two reasons: on the one hand, the variable is less affected by endogeneity issues that may arise in the estimation as a consequence of the possible causal relationship between labor market status and the rational choice of having a child, than the choice of having an elderly relative living in the household. ${ }^{13}$ On the other hand, it captures the increasing relevance of public policies aimed at meeting the increased demand for long-term care and assistance. These considerations are validated by the empirical finding, which shows that having children exhibits an ambiguous and often not significant impact on women's employment, as noted, for example, by Haas et al. (2006), Del Boca and Sauer (2009), and Cipollone and D'Ippoliti (2010).

Thus, overall we assume that the three macro indices developed in the previous section (CD, PPS, and LMC) will exert a direct effect on women's employment and an indirect effect by interacting with two individual characteristics, namely living with a partner (the variable "married") and with a dependent person of old age (the variable "old age"). By contrast, we assume that all the other individual variables included in the analysis exert an effect that is independent from the contextual variables. Specifically, we assume that the impact of education on women's employment is substantially homogeneous across regions. This hypothesis arises from the observation that employment 
levels of the more educated women are similar across Italy's regions, and the reward for human capital investments in terms of employability does not appear to be related to the institutional context (Cipollone and D'Ippoliti 2010).

\section{Model's Results}

By using the combined micro-macro dataset, we estimate a two-level random-coefficient probit model of employment. First, this article reports the results of a pooled sample, including both men and women, and then separately for women. The interest in applying our multilevel method to the pooled estimation, besides its role as a benchmark, is that it allows us to provide an explanation for the residual difference in the labor market inclusion between women and men that cannot be explained by gender differences in individual characteristics. We explain this systematic residual difference, measured by a dummy variable, "woman," by means of the three macro indicators CD, PPS, and LMC. As explained in the previous section both in the pooled estimation and in that on the female subsample, we also allow for cross-level interactions between the three macro factors and two other individual-level variables, namely the dummy variables "being married" and "living with a dependent person of old age." ${ }^{\text {14 }}$

\section{Pooled Estimation}

The three regional factors exert a significant overall impact on the individuals' likelihood of being employed, though their specific contribution has been rather variable over the 30-year period, as shown in Table A. 2 in Appendix. Regional economic growth (LMC) seems to be the factor that is consistently more conducive to higher employment, as measured by its marginal effects (that is, the average change in the individuals' probabilities stemming from a 1 percent change in the independent variable). By contrast, CD and PPS exhibit a couple of problematic sign reversals and their coefficients are not always statistically significant. These ambiguous results will, however, decline when proceeding with separate estimates, thus supporting the view that gender-specific processes may exist and that separate genderspecific estimations are necessary. 


\section{Table 3}

Details of the Second Level: The Influence of the Macro Factors on the Coefficient of the Dummy Variable "Woman"

\begin{tabular}{lcccc}
\hline & 1977 & 1986 & 1995 & 2004 \\
\hline Woman: St.Dev. & 0.255 & 0.295 & 0.257 & 0.296 \\
& $0.016^{* * *}$ & $0.032^{* * *}$ & $0.033^{* * *}$ & $0.022^{* * *}$ \\
Regional Intercept & -0.855 & -0.576 & -0.541 & -0.762 \\
& $0.072^{* * *}$ & $0.157^{* * *}$ & $0.114^{* * *}$ & $0.103^{* * *}$ \\
CD & 0.016 & 0.125 & 0.106 & 0.260 \\
& -0.026 & $0.028^{* * *}$ & $0.032^{* * *}$ & $0.035^{* * *}$ \\
PPS & -0.089 & -0.054 & -0.133 & 0.096 \\
& $0.045^{* *}$ & $0.041^{*}$ & $0.061^{* *}$ & $0.026^{* * *}$ \\
LMC & -0.183 & -0.240 & 0.006 & 0.172 \\
& $0.063^{* * *}$ & $0.058^{* * *}$ & -0.019 & $0.013^{* * *}$ \\
\hline
\end{tabular}

${ }^{* * *}$ statistically significant at $1 \%$; ${ }^{* *}$ statistically significant at $5 \%$; statistically significant at $10 \%$.

Source: Elaboration on SHIW (various years, sample restricted to age bracket [25-54]) and various sources (see Table 1).

As predicted by most of the literature, the impact of individual characteristics is highly relevant. Living with a person of old age, in particular, negatively affects the probability of being employed, while being married appears to be irrelevant. Education exerts the usual positive impact on the likelihood of being employed, though with a decreasing historical trend in the period considered. ${ }^{15}$ As mentioned, the main reason for estimating a pooled regression is to investigate the dummy variable "woman." In our estimates, its impact remains significantly negative during the whole period (with a marginal effect ranging between -50 percent and -70 percent), even after controlling for individual and macro variables.

Thus, women exhibit a systematically lower probability of being employed that is not explained by the other mentioned factors. Table 3 shows the detailed breakdown of the impact of the three macro factors, and of the regional intercepts on the coefficient of the dummy variable, "woman." These second-level impacts measure the 
extent to which our macro factors are able to explain these crossregional differences that individual characteristics are not able to account for.

As it emerges, the impact of being a woman on the likelihood of being employed is significantly correlated to macro factors, as shown by the significance of CD, PPS, and LMC in several estimations. Ceteris paribus the negative impact on employment of being a woman is partly mitigated in the regions with a larger index of $\mathrm{CD}$, as shown by the positive coefficients for the interaction between being a woman and CD. It is noteworthy that this interaction effect seemed to have strengthened over time, as shown by the increasing magnitude of its coefficient (from 0.016 in 1977 to 0.260 in 2004). Instead, the coefficients of the interaction terms between being a woman and PPS and LMC are consistently less significant, and are unstable over the period of analysis. They were negative in 1977 and became significantly positive in 2004. A region's unobserved heterogeneity (that is residual region-specific variance that the three indices are unable to explain) is significant and large throughout the period. Thus, the explanation for women's disadvantage in the labor market may require the consideration of even further variables in the analysis.

\section{Estimation on Women}

The results of the estimations on the women's sample are listed in Table 4 . They are substantially different from those for the population average (as summarized by the pooled sample). Throughout the period, education is confirmed as the strongest individual characteristic determining women's employment. Its impact is larger than that found in the pooled sample and it is monotonically increasing over time.

As opposed to what was found in the pooled sample, being married significantly decreases women's probability of being employed and its impact varies between -9.6 percent in 1986 and -27 percent in 2004 . By contrast, the impact of living with a person of old age significantly changed during the period. Interestingly, it exhibits a positive coefficient in 1977, and increasingly negative coefficients in the following years. This gradual shift may depend on the changing family structure 


\section{Table 4}

Estimation on Women: Two-Level Probit Regression, Marginal Effects

\begin{tabular}{|c|c|c|c|c|}
\hline & 1977 & 1986 & 1995 & 2004 \\
\hline Level 1 units & 2,863 & 4,928 & 5,274 & 4,476 \\
\hline Level 2 units & 19 & 19 & 19 & 19 \\
\hline Log-likelihood & $-8,348,920.7$ & $-6,689,871.7$ & $-7,683,671.9$ & $-7,786,147.7$ \\
\hline Primary education & $\begin{array}{l}0.528 \\
0.122^{* *}\end{array}$ & $\begin{array}{r}-0.16 \\
0.12 * *\end{array}$ & $\begin{array}{l}0.143 \\
0.232\end{array}$ & $\begin{array}{l}1.101 \\
0.337^{* *}\end{array}$ \\
\hline $\begin{array}{l}\text { Lower secondary } \\
\text { education }\end{array}$ & $\begin{array}{l}1.283 \\
0.192^{* *}\end{array}$ & $\begin{array}{l}0.705 \\
0.170^{* * * *}\end{array}$ & $\begin{array}{l}0.595 \\
0.28 \text { ** }\end{array}$ & $\begin{array}{l}1.437 \\
0.371^{* *}\end{array}$ \\
\hline $\begin{array}{l}\text { Upper secondary } \\
\text { education }\end{array}$ & $\begin{array}{l}1.542 \\
0.13 * *\end{array}$ & $\begin{array}{l}1.011 \\
0.160^{* * *}\end{array}$ & $\begin{array}{l}1.109 \\
0.29 * *\end{array}$ & $\begin{array}{l}1.857 \\
0.372^{* *}\end{array}$ \\
\hline College education & $\begin{array}{l}2.062 \\
0.20 T^{\text {*** }}\end{array}$ & $\begin{array}{l}1.152 \\
0.166 \text { ** }\end{array}$ & $\begin{array}{l}1.463 \\
0.311^{* *}\end{array}$ & $\begin{array}{l}2.306 \\
0.368 \text { ** }\end{array}$ \\
\hline Regional variance & $\begin{array}{l}-2.703 \\
0.615^{* *}\end{array}$ & $\begin{array}{r}-0.122 \\
0.142\end{array}$ & $\begin{array}{c}-5.198 \\
0.591^{* *}\end{array}$ & $\begin{array}{c}-1.443 \\
0.366 * *\end{array}$ \\
\hline Married & $\begin{array}{c}-0.225 \\
\quad 0.079^{* *}\end{array}$ & $\begin{array}{c}-0.096 \\
0.070^{\text {*** }}\end{array}$ & $\begin{array}{c}-0.221 \\
\quad 0.048^{* *}\end{array}$ & $\begin{array}{l}-0.063 \\
\quad 0.029 \text { ** }\end{array}$ \\
\hline Old-age co-living & $\begin{array}{l}0.164 \\
0.064^{* *}\end{array}$ & $\begin{array}{r}-0.147 \\
0.073^{* * *}\end{array}$ & $\begin{array}{l}-0.16 \\
\quad 0.041^{* *}\end{array}$ & $\begin{array}{l}-0.393 \\
0.045 * *\end{array}$ \\
\hline CD & $\begin{array}{l}0.092 \\
0.016^{* *}\end{array}$ & $\begin{array}{l}0.018 \\
0.014 *\end{array}$ & $\begin{array}{l}0.041 \\
0.024 *\end{array}$ & $\begin{array}{l}0.385 \\
0.020^{* * * *}\end{array}$ \\
\hline PPS & $\begin{array}{l}-0.147 \\
0.026^{* *}\end{array}$ & $\begin{array}{l}-0.095 \\
0.029 \text { *** }\end{array}$ & $\begin{array}{c}-0.125 \\
\quad 0.042^{* *}\end{array}$ & $\begin{array}{l}-0.120 \\
0.014 \text { ** }\end{array}$ \\
\hline LMC & $\begin{array}{l}-0.104 \\
0.041^{* * *}\end{array}$ & $\begin{array}{l}-0.036 \\
0.037^{* *}\end{array}$ & $\begin{array}{l}0.07 \\
0.02 \text { *** }\end{array}$ & $\begin{array}{l}0.065 \\
0.004^{* * *}\end{array}$ \\
\hline
\end{tabular}

*statistically significant at $10 \%$; ** statistically significant at $5 \%$; ****statistically significant at $1 \%$. Other control variables: real wealth, age, age squared, urban size ( 4 dummy variables).

Source: Elaboration on SHIW (various years, sample restricted to age bracket [25-54]) and various sources (see Table 1).

and specifically on the shift from extended families-where it may be assumed that the elderly were actively involved in home production and unpaid labor (for example, care for the children) - to the nuclear family, where the elderly come back as recipients of informal longterm care (see Leitner 2003; Saraceno 2010). 
On the whole, the results concerning the macro indicators are consistent with some of the hypotheses listed in the previous section, though with relevant exceptions. Specifically, CD exerts an unambiguously positive effect on women's employment. It is the most relevant contextual variable and at the end of the period (2004) it peaks reaching a +38.5 percent marginal impact. Thus our first hypothesis $\left(\mathrm{H}_{1}\right)$ is confirmed. Interestingly, the index of PPS systematically reduces women's probability to be employed, thus confirming $\mathrm{H}_{2}$ and contradicting the idea that women's employment benefits from the extension of the service sector rather than from social policy. Finally, the impact of LMC, which was systematically positive in the pooled sample, is now ambiguous: negative in 1977 and 1986, and positive in 1995 and 2004. We can thus reject the hypothesis that women's employment unambiguously responds to business cycles, and that it does respond more than men's (rejecting $\mathrm{H}_{3}$ ).

Concerning the details of the second level (reported in Appendix), we find that the three macro indices exert a large and statistically significant impact on the coefficient of the two dummy variables on many occasions. Thus, the crucial hypothesis $\mathrm{H}_{4}$ is confirmed.

In particular, the index of gender-aware civic development exerts an increasingly negative impact on the coefficient of being married, becoming statistically significant at the end of the period (thus providing some partial evidence in favor of $\mathrm{H}_{5}$ ). Hence it may be said that while women's formal employment is increasingly affected by the reduction of gender discrimination and the development of a genderaware culture, the dynamics of unpaid work in the household and the unequal sharing of family chores are not well explained by our model and deserve further investigation.

Finally, macro factors are also relevant in determining the impact on women's employment of informal care burdens, as summarized by the dummy variable "living with a dependent person of old age." As mentioned, the latter variable was positively related to women's probability of being employed in 1977, and negatively since 1986. Thus, the interpretation of the relative interaction terms is more difficult. However, the PPS appears to be positively correlated with the variable's coefficient in 1977 and 1995, and negatively in 1986. As a 
result, we can reject the hypothesis $\mathrm{H}_{6}$, that regions with more progressive social policies in terms of public services for the reconciliation of work and family life unambiguously alleviate the negative effect of housework and family-related responsibilities on women's employment, even though this appears to be true half of the time. In conclusion, this aspect-not totally unrelated to the previous ambiguity on households' division of labor-deserves further investigation as well.

\section{Concluding Remarks}

Between the late 1970 s and the early years of the $21^{\text {st }}$ century, women became more and more represented among the employed population in all developed countries. This phenomenon stimulated a widening literature. In the field of mainstream economics, this literature can be divided into two related, though parallel, strands: one dealing with the impact of individual and household-level characteristics, and the other with the role of macro factors. So far, these empirical analyses have captured two types of effects on women's inclusion in the labor market: (i) the impact of the individual factors, taking macro factors as constant; or (ii) the other way around, that is, the impact of macro factors assuming micro factors as constant. Few works in this literature consider the interactions between these two levels by analyzing some specific individual and macro factors. However, they neglect the role of possible sources of unobserved heterogeneity that may arise by the omission of other relevant macro factors. This work has provided empirical evidence against the robustness of these approaches, while recognizing that some insights therein advanced may indeed prove useful.

We estimated a multilevel model of the determinants of women's employment in Italy. Italy provides a very convenient framework for such an analysis because the increasing rate of women's employment has been accompanied by a persistent level of territorial heterogeneity both in terms of the institutional context and the economic position of women.

We showed that macro and individual factors are both relevant for predicting women's employment, and that they significantly interact. 
Indeed, the impact of some individual and household factors and the systematic residual differences in the labor market inclusion of women and men depend on the institutional setting. This study considered, in particular, the role of gender-based discrimination and cultural norms (proxied by the macro index $\mathrm{CD}$ ), of progressive social policies (in terms of public services for the reconciliation of work and family life), and their interaction with the housework burden on women.

Such factors explain a large part of the historical increase of women's employment in Italy, but still a substantial region-specific unobserved heterogeneity remains significant in all regressions, implying that the consideration of other determinants (besides the wide array of factors considered here) may be necessary. In particular, other possible interactions between individual and macro characteristics may be in place. Thus, further analysis is necessary, for example, to study the roles and determinants of the gender division of housework and fertility, and of their impact on women's employment.

\section{Notes}

1. Of course, the two parallel strands could more accurately be described as linked, rather than separated, if a broader literature was considered.

2. Recent works provide evidence of the existence of such an interaction between the two micro and macro levels. For example, Lefebvre et al. (2009) find that the provision of childcare facilities and subsidies for day-care spaces has a positive impact on women's labor supply, and that this impact is more relevant for the less educated women. Similarly, Gutiérrez-Domènech (2005), studying women's transitions from employment to non-employment after the first birth, finds that in a number of European countries, the impact of education on women's labor supply is likely to depend on the institutional characteristics of the labor market. Goodpaster (2010) finds that in the United States, maternity has had a differentiated impact on women's participation rate, according to a crucial social policy instrument, namely maternity leave regulations.

3. Thus, we take a binary approach to employment: individuals are considered to be employed or not employed. However, we recognize that important issues are also the engagement in part-time work or the distinction between unemployed and inactive population. Moreover, we specifically focus on women's employment rather than women's participation for several reasons. On the one hand, we maintain that among the key labor market 
indicators, the employment rate constitutes the best index of labor market dynamics and functioning. On the other hand, in terms of the reciprocal influence of the key labor market indicators, the employment rate can play the major role. Finally, Italy lags well behind the Lisbon target in terms of women's employment rate, and this index thus constitutes a major priority for economic policy.

4. Surprisingly, it is found that in developed countries, men's employment responds more to both positive and negative macroeconomic fluctuations because, due to horizontal segregation, women are often employed in more stable industries and/or safer-though low-paid-jobs (e.g., as civil servants).

5. See, among the others, Putnam et al. (1993).

6. The choice of a nine-year intertemporal window was directed by issues of data availability and the widespread evidence that changes in labor market inclusion and labor supply take some time to occur, mostly depending on variables that only slowly respond to the business cycle and policy innovations (for example, concerning education, taxation, culture, allocation of time, and household's resources).

7. Moreover, in the second (aggregate) level, there are only 20 observations corresponding to the Italy's 20 regions, and consequently, a large number of independent variables would not be identified.

8. The variable information, synthesizing the average households' expenditure for periodicals and newspapers, was not available for the year 1977.

9. Bettio (2002) argues that there are pros and cons of gender horizontal occupational segregation, and that from some points of view (for example, the gender pay gap) it should not be considered as a completely negative phenomenon. However, it is considered as such within this analysis becausewhile this research ignores incomes - this study is mainly concerned with its economic dimension as a barrier to fair competition in the labor market, and with its cultural dimension as a factor confirming gender stereotypes.

10. By prominent self-employment this paper means those occupations (namely general practitioner and specialized medical occupations, notary, business consultant, barrister and other law consultants) that enjoy high income and respected social status as a consequence not only of the high skills involved, but also of the public certifications that are necessary to enter the profession. As the issue of these certifications is managed by the very same professional associations, they are able to erect high barriers to entry and are more likely to discriminate against women, as noted by Cipollone and D'Ippoliti (2010).

11. Thus, rotation of factors was adopted by means of the varimax method, which attempts to minimize the number of variables that have a high correlation coefficient on a factor. Three factors were selected, which satisfy the following requirements: exhibiting eigenvalues close to or larger than unity; 
individual contribution to the explanation of the overall variance of the data greater than 10 percent; and cumulative contribution to the explanation of the total variance of the data greater than 60 percent. Within each of these factors, the single indicators are weighted according to the proportion of their cross-region variance explained by the factor. As a result, regions can be scored on each of the factors using the estimated weights.

12. This last term is necessary in so far as observations within groups (within a single region) are more likely to be correlated than observations from different groups (across regions), and failure to control for this secondlevel heterogeneity may lead to inconsistent estimates and misleading inferences.

13. For a rigorous proof of this statement, compare the supporting materials available at the authors' webpage http://w3.uniroma1.it/dippoliti/ pubblicazioni-scientifiche.html.

14. The number of individual variables that are allowed to interact with contextual variables is limited by computational requirements.

15. For the years 1977 and 1986, the variable education was missing for a number of working-age women and men. To address this issue, this research imputed the corresponding value by means of iterative techniques, using the module for Stata called Imputation by Chained Equations (ICE) described by Royston (2004, 2005).

\section{References}

Algan, Y., and P. Cahuc. (2007). "The Roots of Low European Employment: Family Culture?" In NBER International Seminar on Macroeconomics 2005. Eds. C. Pissarides and J. Frenkel, pp. 65-109. Cambridge: MIT Press.

Anderson, P. M., and P. B. Levine. (1999). "Child Care and Mothers' Employment Decisions." NBER Working Paper No. 7058.

Antonopoulos, R. (2009). "The Current Economic and Financial Crisis: A Gender Perspective." The Levy Economics Institute of Bard College Working Paper No. 562.

Apps, P., and A. Rees. (2004). "Fertility, Taxation and Family Policy." Scandinavian Journal of Economics 106(4): 745-763.

Attanasio, O., H. Low, and V. Sanchez-Marcos. (2008). "Explaining Changes in Female Labor Supply in a Life-Cycle Model." American Economic Review 98(4): 1517-1552.

Bardasi, E., and J. C. Gornick. (2003). "Women and Part-time Employment: Workers' Choices' and Wage Penalties in Five Industrialized Countries." In Women in the Labor Market in Changing Economies: Demographic Issues. Eds. B. Garcia, R. Anker, and A. Pinnelli, pp. 209-244. Oxford: Oxford University Press. 
Becker, G. S. (1965). "A Theory of the Allocation of Time." Economic Journal 75(299): 493-517.

Berlinski, S., and S. Galiani. (2007). "The Effect of a Large Expansion of Pre-Primary School Facilities on Preschool Attendance and Maternal Employment." Labor Economics 14(3): 665-680.

Bettio, F. (2002). "The Pros and Cons of Occupational Gender Segregation in Europe." Canadian Public Policy/Analyse de Politiques 28(S1): 65-84.

Boeri, T., D. Del Bocaand, and C. Pissarides. (2005). Women at Work. Oxford: Oxford University Press.

Casadio, E., and P. Palazzi. (2004). "An Index for Sustainable Development." BNL Quarterly Review LVII(229): 185-206.

Cipollone, A., and C. D'Ippoliti. (2010). "Discriminating Factors of Women's Employment." Applied Economics Letters 17(11): 1055-1062.

Del Boca, D. (2002). "The Effect of Child Care and Part Time Opportunities on Participation and Fertility Decisions in Italy." Journal of Population Economics 15(3): 549-573.

Del Boca, D., S. Pasquaand, and C. Pronzato. (2009). "Motherhood and Market Work Decisions in Institutional Context: A European Perspective." Oxford Economic Papers 61(suppl_1): i147-i171.

Del Boca, D., and R. M. Sauer. (2009). "Life Cycle Employment and Fertility Across Institutional Environments." European Economic Review 53(3): 274-292.

Eckstein, Z., and O. Lifshitz. (2009). "Dynamic Female Labor Supply." CEPR Discussion Paper No. 7548.

Fernandez, R. (2007). "Women, Work, and Culture." Journal of the European Economic Association 5(2-3): 305-332.

Fortin, N. (2005). "Gender Role Attitudes and Women's Labor Market Outcomes Across OECD Countries." Oxford Review of Economic Policy 21(3): 416-438.

Fuchs, V. R. (1980). "Economic Growth and the Rise of Service Employment." NBER Working Paper No. 486.

Fuwa, M. (2004). "Macro-Level Gender Inequality and the Division of Household Labor in 22 Countries." American Sociological Review 69: 751-767.

Fuwa, M., and P. N. Cohen. (2007). "Housework and Social Policy." Social Science Research 36(2): 512-530.

Galor, O., and D. N. Weil. (1996). "The Gender Gap, Fertility and Growth." American Economic Review 86(3): 374-387.

Giavazzi, F., F. Schiantarelli, and M. Serafinelli. (2009). "Culture, Policies and Labor Market Outcomes.” NBER Working Paper Series No. 15417.

Goldin, C. (1990). Understanding the Gender Gap: An Economic History of American Women. Oxford: Oxford University Press.

—. (2002). "A Pollution Theory of Discrimination: Male and Female Differences in Occupations and Earnings.” NBER Working Paper No. 8985. 
—. (2006). "The Quiet Revolution that Transformed Women's Employment, Education and Family." American Economic Review 96(2): 1-21.

González, M. J., T. Jurado-Guerrero, and M. Naldini. (2009). "What Made Him Change? An Individual and National Analysis of Men's Participation in Housework in 26 Countries." DemoSoc Working Paper 30. Universitat Pompeu Fabra, Barcelona.

Goodpaster, N. K. (2010). "Leaves and Leaving: The Family and Medical Leave Act and the Decline in Maternal Labor Force Participation." B.E. Journal of Economic Analysis \& Policy 10(1): Art. 6.

Gustafsson, S., and E. Kenjoh. (2008). "The Timing of Maternity." In Social Policies, Labor Markets and Motherhood. Eds. D. Del Boca and C. Wetzles, pp. 182-224. Cambridge: Cambridge University Press.

Gutiérrez-Domènech, M. (2005). "Employment After Motherhood: A European Comparison." Labor Economics 12(1): 99-123.

Haas, B., N. Steiber, M. Hartel, and C. Wallace. (2006). "Household Employment Patterns in an Enlarged European Union." Work, Employment and Society 20(4): 751-771.

ILO (2009). Global Employment Trends for Women. Geneva: International Labor Office.

Jaumotte, F. (2003). "Female Labor Force Participation. Past Trends and Main Determinants in OECD Countries." OECD Economic Studies 37: 52-108.

Kline, P. (2004). An Easy Guide to Factor Analysis. London: Routledge.

Knudsen, K., and K. Wærness. (2007). "National Context and Spouses' Housework in 34 Countries." European Sociological Review 24(1): 97-113.

Kunovich, R. M., and S. Kunovich. (2008). "Gender Dependence and Attitudes Toward the Distribution of Household Labor A Comparative and Multilevel Analysis." International Journal of Comparative Sociology 49(6): 395-427.

Lefebvre, P., P. Merrigan, and M. Verstaete. (2009). "Dynamic Labor Supply Effects of Childcare Subsidies: Evidence from a Canadian Natural Experiment on Low-Fee Universal Child Care." Labor Economics 16(5): 490-502.

Leigh, A. (2010). "Informal care and labor market participation". Labor Economics 17(1): 140-149.

Leitner, S. (2003). "Varieties of Familialism: The Caring Function of the Family in Comparative Perspective." European Societies 5(4): 353-375.

Lundin, D., E. Mörk, and B. Öckert. (2008). "How Far Can Reduced Childcare Prices Push Female Labor Supply?” Labor Economics 15(4): 647-659.

Munasinghe, L., T. Reif, and A. Henriques. (2008). "Gender Gap in Wage Returns to Job Tenure and Experience." Labor Economics 15(6): 12961316.

Nardo, M., M. Saisana, A. Saltelli, S. Tarantola, A. Hoffman, and E. Giovannini. (2005). "Handbook on Constructing Composite Indicators: Methodology and User Guide." OECD Statistics Working Papers No. 2005/3. 
Nicoletti, G., S. Scarpetta, and O. Boylaud. (1999). "Summary Indicators of Product Market Regulation with an Extension to Employment Protection Legislation." OECD Economic Department Working Papers No. 229.

OECD (2004). Benefits and Wages. OECD Indicators. Paris: OECD.

Potestio, P. (2005). "L'occupazione in Italia dal 1970 al 2003." Rivista di Politica Economica XCV(3-4): 245-322.

Powell, L. M. (1998). "Part-Time Versus Full-Time Work and Childcare Costs: Evidence for Married Mothers." Applied Economics 30(4): 503-511.

Putnam, R., R. Leonardi, and R. Y. Nanetti. (1993). Making Democracy Work: Civic Traditions in Modern Italy. Princeton: Princeton University Press.

Royston, P. (2004). "Multiple Imputation of Missing Values." Stata Journal 4(3): 227-241.

—. (2005). "Multiple Imputation of Missing Values: Update of ICE." Stata Journal 5(4): 527-36.

Sánchez-Mangas, R., and V. Sánchez-Marcos. (2008). "Balancing Family and Work: The Effect of Cash Benefits for Working Mothers." Labor Economics 15(6): 1127-1142.

Saraceno, C. (2010). "Social Inequalities in Facing Old-Age Dependency: A Bi-Generational Perspective." Journal of European Social Policy 20(1): 32-44.

Simonazzi, A. (2006). "Care Regimes and National Employment Models." Cambridge Journal of Economics 33(2): 211-232.

Smith, M. (2009). Analysis Note: Gender Equality and Recession. Report for the European Commission-DG Employment, Social Affairs and Equal Opportunities. Rome: EGGE (European Network of Experts on Employment and Gender Equality Issues).

Smith, R. J., and R. W. Blundell. (1986). "An Exogeneity Test for a Simultaneous Equation Tobit Model with an Application to Labor Supply." Econometrica 54(4): 679-686.

Stier, H., and N. Lewin-Epstein. (2007). "Policy Effects on the Division of Housework." Journal of Comparative Policy Analysis 9(3): 235259.

van Damme, M., M. Kalmijn, and W. Uunk. (2008). "The Employment of Separated Women in Europe: Individual and Institutional Determinants." European Sociological Review 25(2): 183-197.

van der Lippe, T., and L. van Dijk. (2002). "Comparative Research on Women's Employment." Annual Review of Sociology 28: 221-241.

Voicu, M., B. Voicu, and K. Strapcova. (2008). "Housework and Gender Inequality in European Countries." European Sociological Review 25(3): 365-377. 


\section{Supporting Information}

Additional supporting information may be found in the online version of this article:

Figure A.1: Long-Run Evolution of the Civic Development Index (CD) Figure A.2: Long-Run Evolution of the Civic Development Index, mean values by geographical area

Figure A.3: Long-Run Evolution of the Private Provision of Services Index (PPS)

Figure A.4: Long-Run Evolution of the Private Provision of Services Index, mean values by geographical area

Figure A.5: Long-Run Evolution of the Local Macroeconomic Conditions Index

Figure A.6: Long-Run Evolution of the Local Macroeconomic Conditions Index (LMC), mean values by geographical area

Table A.1: Descriptive Statistics for the Original Macroeconomic Variables and the Aggregated Indexes.

Table A.2: Pooled Estimation: Two-Level Probit Regression, Marginal Effects

Table A.3: Details of the Second Level: The Influence of the Macro Factors on the Coefficient of the Dummy Variable "Being Married"

Table A.4: Details of the Second Level: The Influence of the Macro Factors on the Coefficient of the Dummy Variable "Co-Living with an Old-Aged Dependent"

Please note: Wiley-Blackwell is not responsible for the content or functionality of any supporting materials supplied by the authors. Any queries (other than missing material) should be directed to the corresponding author for the article. 